\title{
Legal aspects of open disclosure II: attitudes of health professionals — findings from a national survey
}

\author{
David M Studdert, Donella Piper and Rick ledema
}

工 nterest in the practice of open disclosure (OD) - candid communication between health professionals and patients following adverse events in health care - has exploded internationally in the past decade. ${ }^{1-5}$ Australia has emerged as an international leader in this area. A national Open Disclosure Standard ("the Standard"), designed to facilitate open communication about adverse events, was endorsed by the health ministers in $2003 .{ }^{6}$

A recent evaluation of the Standard's implementation in 21 pilot sites demonstrated a reasonable degree of enthusiasm for OD among both patients and clinicians. ${ }^{7}$ However, legal concerns were apparent. A number of health professionals voiced uncertainty about the implications of OD for medicolegal risk and liability insurance coverage. ${ }^{7}$ In addition, the Standard's rollout was initially stalled by concerns from state government insurers about the fiscal and litigation consequences of offering expressions of regret to patients who had been harmed. Survey research in the United States has identified fear of increased liability as one of the main reasons doctors are reluctant to embrace OD, ${ }^{4,8,9}$ but no previous research has addressed this question in Australia.

We surveyed a sample of health professionals at the forefront of OD practice in Australia. Our main objective was to gauge the perceived importance of medicolegal fears as a barrier to OD. Because some existing state laws - chiefly, apology and qualified privilege laws - may protect information conveyed in OD from subsequent use in legal proceedings, the survey also elicited views on the extent to which such laws supported OD practice.

\section{METHODS}

\section{Sample}

OD processes are a relatively new feature of health care delivery systems. We expected limited knowledge of laws related to them within any random sample of health professionals. We therefore targeted the survey at a small group of recognised experts - individuals with direct experience with actual OD communications. In addition, because

\section{ABSTRACT}

Objective: To assess the attitudes of health care professionals engaged in open disclosure (OD) to the legal risks and protections that surround this activity.

Design and participants: National cross-sectional survey of 51 experienced OD practitioners conducted in mid 2009.

Main outcome measures: Perceived barriers to OD; awareness of and attitudes towards medicolegal protections; recommendations for reform.

Results: The vast majority of participants rated fears about the medicolegal risks (45/51) and inadequate education and training in OD skills (43/51) as major or moderate barriers to OD. A majority (30/51) of participants viewed qualified privilege laws as having limited or no effect on health professionals' willingness to conduct OD, whereas opinion was divided about the effect of apology laws (state laws protecting expressions of regret from subsequent use in legal proceedings). In four states and territories (Western Australia, South Australia, Tasmania and the Northern Territory), a majority of participants were unaware that their own jurisdiction had apology laws that applied to OD. The most frequent recommendations for legal reform to improve OD were strengthening existing protections (23), improving education and awareness of applicable laws (11), fundamental reform of the medical negligence system (8), and better alignment of the activities of certain legal actors (eg, coroners) with OD practice (6).

Conclusions: Concerns about both the medicolegal implications of OD and the skills needed to conduct it effectively are prevalent among health professionals at the leading edge of the OD movement in Australia. The ability of current laws to protect against use of this information in legal proceedings is perceived as inadequate.

MJA 2010; 193: 351-355

laws that bear on OD practice are chiefly state laws, we sought to include in the sample participants from all eight states and territories, roughly in proportion to population size.

Participants were identified in three principal ways. First, staff from the Australian Commission on Safety and Quality in Health Care (ACSQHC) provided names of OD leaders from across the country. Second, we contacted directors of patient safety and quality in state departments of health and requested names of people recognised as leaders of OD practice in that state. Third, we used snowball sampling: at the completion of each survey, we asked participants whether they knew of counterparts at other health care institutions who were knowledgeable about and experienced with OD. Our target sample size was 50 participants.

\section{Survey instrument}

The instrument asked participants to rate the importance of 11 potential barriers to OD on a four-point Likert scale (from "not a barrier" to "major barrier"). A barrier termed "fears about medicolegal risks" was presented midway down the list.

Next, participants rated their views about the extent to which apology laws and qualified privilege laws, respectively, made health professionals "more willing to conduct full and frank open disclosure". Apology laws were defined as "laws that protect apologies or expressions of regret from later use in legal proceedings". Qualified privilege laws were defined as "laws that protect certain types of information related to quality improvement efforts, such as information that comes out of the work of a hospital's quality assurance committee and incident reports, from use in legal proceedings". Participants rated their willingness on a five-point Likert scale (from "not at all" to "much more willing"). These questions probed participants' general views on the effectiveness of apology and qualified privilege laws, untethered to the legal situation in any given jurisdiction. Subsequent questions ascertained participants' knowledge of prevailing laws in their own jurisdiction. 
Finally, the instrument elicited participants' opinions about whether legal reforms are needed to "help ensure the free flow of information between providers and patients about adverse events", and if so, what those reforms should be. Law reform recommendations were recorded as free text. We pilottested a draft version of the instrument on three people who met the sampling criteria.

\section{Survey administration}

One of us (DP) administered the survey by telephone between April and June 2009. Completion times varied in duration from 14 minutes to 65 minutes, with a median of 25 minutes.

We approached a total of 83 people, of whom 20 did not have direct experience with OD practice and thus were ineligible to participate. Of the rest, nine did not respond to telephone and email requests to be surveyed and three refused. The remaining 51 people agreed to participate and completed the survey (a response rate of 81\% [51/63] among eligible participants)

1 Profile of participants $(n=51)$

\begin{tabular}{lr} 
Characteristic & $n$ \\
\hline State/territory of residence & 10 \\
New South Wales & 8 \\
Queensland & 7 \\
Victoria & 7 \\
Western Australia & 6 \\
South Australia & 5 \\
Australian Capital Territory & 5 \\
Northern Territory & 3 \\
Tasmania & \\
Job title & 14 \\
Risk manager & 7 \\
Director/manager of clinical & \\
governance & 7 \\
Director of medical/clinical service & 6 \\
Manager consumer services/ & \\
complaints & 5 \\
Patient safety officer & 5 \\
Medical specialist & 3 \\
Director of nursing & 24 \\
Other & \\
Profession & \\
Doctor & \\
Nurse & \\
Nurse/lawyer & \\
Other & \\
& \\
&
\end{tabular}

roughly equally divided as to whether they made participants much more or more willing to conduct OD (19/51); somewhat more willing (14/51); or not more willing (18/51). Among those in the third group, a majority (14/18) indicated that lack of awareness of apology laws was the key inhibiting factor. With respect to qualified privilege laws, 30 participants viewed them as having limited or no effect on OD. The leading reason (12/ 30) given for lack of effect was that the laws did not cover situations or types of information pertinent to OD activities. As one participant put it, "I don't think they have any bearing on open disclosure"; another said, "We have not tied in qualified privilege with the open disclosure process at all. So for our hospital the impact has been nil, not positive or negative."

\section{Knowledge of legal protections}

Although all states have apology laws that may apply to OD communications, knowledge of that fact was limited, except among participants from New South Wales and the Australian Capital Territory (Box 4). Unlike the general questions about perceived efficacy described above, this set of questions elicited knowledge of the laws actually in force in the participants' jurisdiction. A majority of participants in four states and managers of consumer services $(6 / 51)$ or patient safety officers (5/51).

\section{Barriers}

Two perceived barriers to OD stood out (Box 2): most participants cited fears about medicolegal risks (45/51) and inadequate education and training in OD (43/51) as major or moderate barriers. With mean Likert scores of 3.2 and 3.4, respectively (" 3 " representing a moderate barrier and " 4 " a major barrier), the perceived importance of each of these barriers was significantly greater than any of the other barriers posed $(P<0.001)$.

Four other factors were cited as a major or moderate barrier by about a third of participants: time constraints (18/51), fear of scaring patients (18/51), advice from liability insurers (18/51) and advice from clinical leaders (16/51). Only six participants viewed cost as a major or moderate barrier.

\section{Perceived efficacy of legal protections}

Opinion was mixed over the efficacy of apology laws and qualified privilege laws as tools for promoting OD (Box 3). With respect to apology laws, participants were

\section{Perceived importance of potential barriers to open disclosure (OD) $(n=51)$}

\begin{tabular}{|c|c|c|}
\hline Barrier & $\begin{array}{l}\text { Major or } \\
\text { moderate } \\
\text { barrier, } n\end{array}$ & $\begin{array}{c}\text { Mean } \\
\text { (median) } \\
\text { score* }^{\star}\end{array}$ \\
\hline $\begin{array}{l}\text { Fears about } \\
\text { medicolegal risk }\end{array}$ & 45 & $3.2(3)$ \\
\hline $\begin{array}{l}\text { Inadequate education } \\
\text { and training in OD } \\
\text { skills }\end{array}$ & 43 & $3.4(4)$ \\
\hline Time constraints & 18 & $2.2(2)$ \\
\hline Fear of scaring patients & 18 & $2.2(2)$ \\
\hline Cost concerns & 6 & $1.5(1)$ \\
\hline \multicolumn{3}{|l|}{ Advice from: } \\
\hline Liability insurers & 18 & $2.1(2)$ \\
\hline Clinical leaders & 16 & $2.0(2)$ \\
\hline $\begin{array}{l}\text { Executives (eg, } \\
\text { hospital CEO) }\end{array}$ & 7 & $1.7(2)$ \\
\hline $\begin{array}{l}\text { Professional bodies } \\
\text { (eg, college) }\end{array}$ & 6 & $1.7(1.5)$ \\
\hline Risk managers & 3 & $1.4(1)$ \\
\hline
\end{tabular}


3 Perceived effect of existing qualified privilege and apology laws on health professionals' willingness to conduct full and frank open disclosure (OD) $(n=51)$
Perceived effect on OD behaviour

More willing/much more willing

Somewhat more willing

Not much more willing/not at all willing

Lack of awareness of law

Lack of confidence in law's protective value

Don't know, no experience with them*

Insufficient connection to OD

Other reason

* These responses came from four participants in the Northern Territory, where qualified privilege laws exist but there are currently no health care entities designated under the legislation to enjoy this privilege.

territories (Western Australia, South Australia, Tasmania and the Northern Territory) incorrectly said their jurisdiction did not have an apology law pertaining to $\mathrm{OD}$, or were unsure whether it did. Overall, one in five participants (10/51) indicated that their jurisdiction did not have an apology law when it did, and one in four (14/51) did not know the answer.

The survey also asked participants whether their state had qualified privilege laws "that cover the contents of open disclosure conversations". Only three participants (one from Victoria, two from Queensland) indicated that that their state did; 41 participants said their state did not; and six participants were unsure. Responses to this question are difficult to evaluate. All jurisdictions except NT have qualified privilege statutes, but their relationship to OD is unclear. ${ }^{11} \mathrm{~A}$ negative response may therefore have reflected participants' lack of awareness of the existence of the law or an opinion that the law did not relate to OD. In short, there was no correct answer against which to benchmark responses.

\section{Law reforms}

All but four participants recommended law reforms. The most frequent recommendations pertained to bolstering existing protections by strengthening or clarifying them (23/47), particularly qualified privilege laws (Box 5). The other leading recommendations were: improving education and awareness of existing laws (11/47); fundamental reform of the medical negligence system (8/ 47), including introduction of no-fault compensation for medical injuries (6/47); and better alignment of the activities of various legal actors, such as coroners and health
Apology laws, $n$ Qualified privilege laws, $n$

$\begin{array}{cc}19 & 13 \\ 14 & 8 \\ 18 & 30 \\ 14 / 18 & 3 / 30 \\ 2 / 18 & 5 / 30 \\ 0 / 18 & 4 / 30 \\ 0 / 18 & 12 / 30 \\ 2 / 18 & 6 / 30\end{array}$

complaints commissioners, with the objectives and culture of OD (6/47).

\section{DISCUSSION}

Our survey of health professionals detected considerable apprehension about the liability implications of engaging in OD. Fear of medicolegal consequences, alongside inadequate education and training, was perceived as the leading barrier to OD. More than twothirds of participants rejected, or only tepidly supported, the proposition that the willingness of health professionals to conduct OD is enhanced by existing laws that protect the information from use in legal proceedings.

Our findings resonate with those from previous US studies that have highlighted concerns about OD's medicolegal repercussions as a major obstacle to its uptake., ${ }^{1,4,8}$ For example, in Lamb and colleagues' 2002 survey of hospital-based risk managers, fear of malpractice exposure was the most fre- quently cited barrier to OD, with $77 \%$ of participants identifying it. ${ }^{8}$ Our study found similarly high levels of concern. The other prominent barrier we identified was a lack of education and training in how to conduct OD (an option not presented to respondents in the survey by Lamb and colleagues). Policymakers have recognised the need to provide clinicians with appropriate training in $\mathrm{OD}^{12,13}$ and educational programs are now being developed and rolled out in several states. ${ }^{12,14,15}$

In theory, health professionals' fears about the legal consequences of $\mathrm{OD}$ could be mitigated by laws that prohibit use of the information for inculpatory purposes. To the extent that such protections exist in Australia, apology laws and qualified privilege laws are the main sources. ${ }^{11}$ In practice, however, several factors may undercut the ability of such laws to promote OD. First, the laws may have limited applicability in the OD context. Second, whatever protections they provide may be too weak to assuage medicolegal concerns. Third, health professionals may not understand or be aware of legal protections. Responses to our survey suggest that all three factors are at work.

Ignorance about protective laws was evident in responses to questions about apology laws. Among the third of participants who said that apology laws were not an effective way of encouraging $\mathrm{OD}$, the leading reason given was "lack of awareness" of these protections. Even more compelling was the finding that participants themselves, despite being leaders of OD in Australia, displayed uncertainty and confusion about the existence of apology laws in their own jurisdictions. Every state and territory has an apology law that should cover expressions
4 Participants' responses regarding whether their jurisdiction has a law that protects apologies made in open disclosure

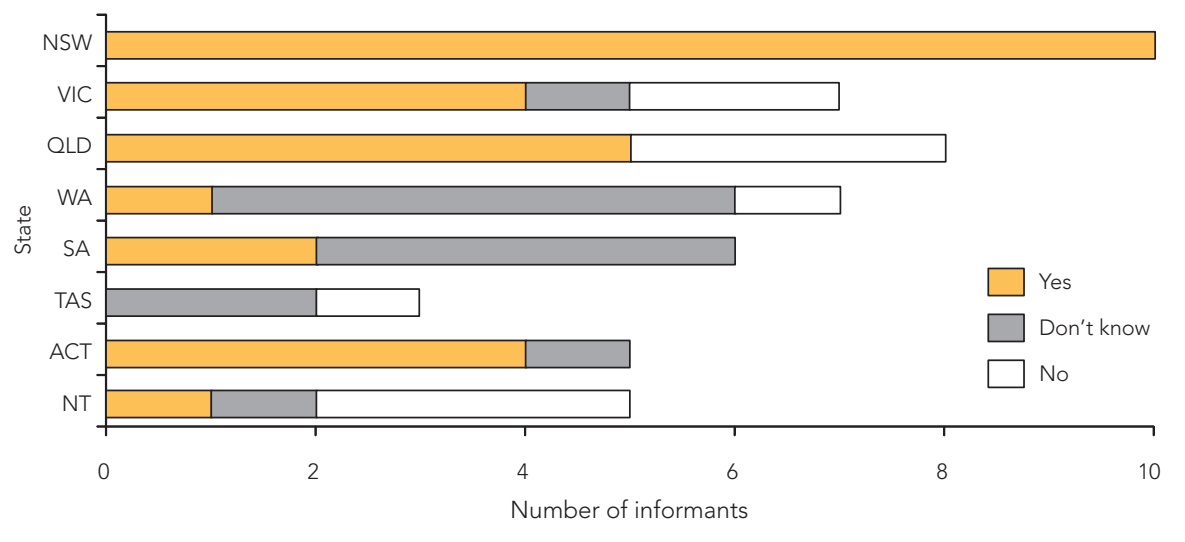




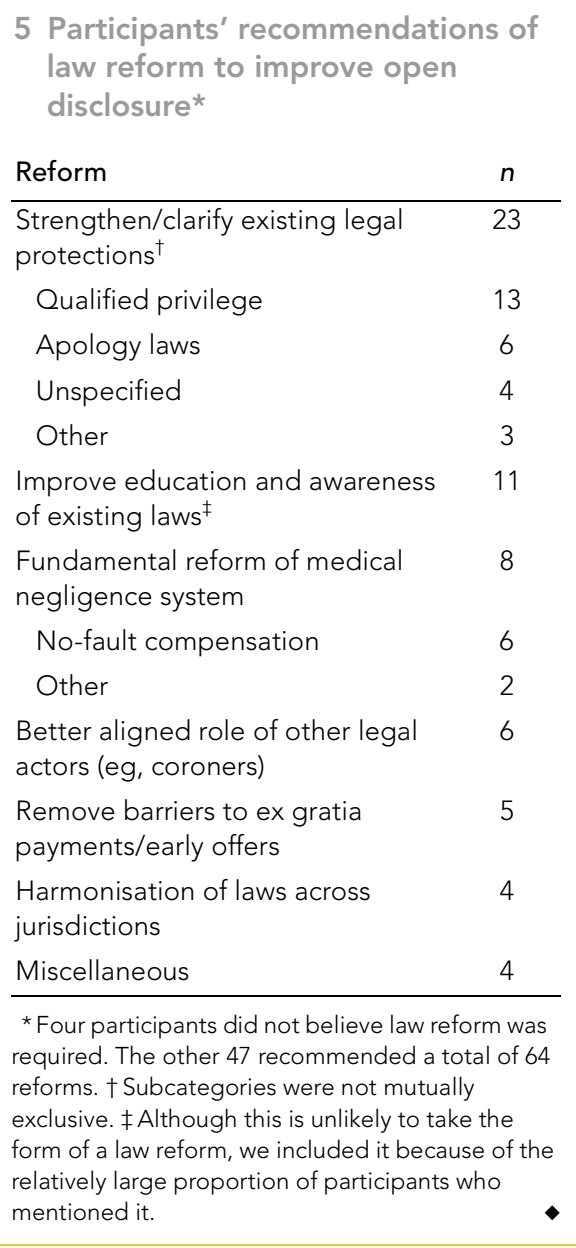

of regret made in OD communications. ${ }^{11}$ Knowledge of this was excellent among participants from NSW and the ACT, fair among participants from Victoria and Queenslands, and poor among participants from the other states and territories.

Many participants were sceptical about the relevance and applicability of qualified privilege laws to OD. A majority (30/51) said that such laws do not encourage OD, and the leading reason given for this lack of effect was that the laws had little or no applicability to OD conversations. The analysis of OD-related laws in the previous issue of the Journal ${ }^{11}$ suggests this view is correct; the broad conclusion of that analysis was that existing legal protections of information conveyed in OD are quite weak. That conclusion matches the perspective of many survey participants, nearly half of whom recommended law reforms designed to strengthen existing protections, particularly qualified privilege.

Another popular suggestion for law reform was education of health professionals about prevailing protections (although, strictly speaking, this is probably not a law reform measure). The benefits of that strategy in the absence of changes to the underlying laws are highly questionable: according to the legal analysis, ${ }^{11}$ an accurate picture of protections under existing laws will be cold comfort. Eight participants said that radical reform of the current negligence system was needed; their concern was that an environment conducive to OD is unattainable against the backdrop of the current adversarial system. This argument joins others for "no blame" systems of compensation for medical injury. ${ }^{16,17}$ Finally, six participants lamented the influence of other legal actors, particularly coroners, whose approach to death investigations was perceived as working at cross-purposes to the culture of OD.

Our study has several limitations. First, the sample size was small, a constraint that stemmed from our focus on experts in an area in which there are relatively few. Second, we did not probe the veracity of participants' concerns, nor did we study the reasonableness of their medicolegal fears. New evidence from a US university health system suggests that an aggressive program of OD does not necessarily worsen a health care institution's liability experience, and may even reduce the incidence and cost of negligence claims. ${ }^{18}$ Third, our non-random recruitment method may have led to a degree of "clustering" in responses, particularly among participants who knew each other. Fourth, clinicians may be quick to cite medicolegal issues as a barrier to OD when what actually chills their interest is a complicated mix of factors, including reputational concerns and fundamental human instincts to avoid conflict. ${ }^{19,20}$ Fifth, the poor understanding some participants had about prevailing laws in their own jurisdiction casts doubt on how informed their responses were to more general questions about the effect of these types of laws on OD practice. Sixth, the survey focused on measuring attitudes to medicolegal factors, not the degree to which those attitudes actually inhibit conduct of OD. Despite their concerns, many health professionals may still forge ahead with OD out of a firm belief that it should be done.

There is broad consensus today that OD following adverse events in care is the right thing to do. ${ }^{1,21}$ Clinicians who have not yet embraced the practice are likely to face mounting external pressures to do so. Certain relatively intractable difficulties with expanding OD loom large - for example, clinicians' concerns about their reputation, natural human instincts to avoid "hard" conversations and admissions of failure, and deeply entrenched aspects of medical culture. $^{1,9,13,21}$ Ironically, the main findings of our study — that medicolegal fears and lack of appropriate OD training are at the top of the list of perceived barriers to OD in Australia - should give OD proponents cause for optimism, as both obstacles should be at least partly fixable. Legal regimes can be reformed to reassure health professionals who are open with patients about adverse events that their forthrightness will not be turned against them. With such reforms in place, educational efforts could then emphasise both the skills needed to conduct OD effectively and the strength of prevailing legal protections.

\section{ACKNOWLEDGEMENTS}

Our study was funded by the ACSOHC. Tom Gallagher provided helpful comments on an earlier draft of the manuscript.

\section{COMPETING INTERESTS}

David Studdert is the chief investigator of an Australian Research Council (ARC) linkage grant in which he is partnering with the Health Services Commissioner of Victoria and Avant Mutual to study complaints and claims regarding informed consent. Most of the project costs are met by the ARC, but Avant Mutual also contributes to the project budget. Donella Piper was contracted by the ACSOHC in 2008 to advise on the development of a consumer engagement strategy, and received reimbursement from the ACSOHC for related travel costs. Rick ledema was paid consultancy fees by the ACSOHC for three research projects relating to $\mathrm{OD}$.

\section{AUTHOR DETAILS}

David M Studdert, LLB, ScD, MPH, Professor and Federation Fellow ${ }^{1}$

Donella Piper, LLB, PhD, LLM, Research Fellow² Rick ledema, PhD, Professor of Organisational Communication, and Director ${ }^{2}$

1 Melbourne School of Population Health and Melbourne Law School, University of

Melbourne, Melbourne, VIC.

2 Centre for Health Communication, Faculty of Arts and Social Sciences, University of

Technology Sydney, Sydney, NSW.

Correspondence: d.studdert@unimelb.edu.au

\section{REFERENCES}

1 Gallagher T, Studdert DM, Levinson W. Disclosing harmful medical errors to patients: recent developments and future directions. $N$ Engl J Med 2007; 356: 2713-2719.

2 Mazor KM, Simon SR, Yood RA, et al. Health plan members' views about disclosure of medical errors. Ann Intern Med 2004; 140: 409-418. 
3 Blendon RJ, DesRoches CM, Brodie M, et al. Views of practicing physicians and the public on medical errors. N Engl J Med 2002; 347: 1933-1940.

4 Gallagher $T H$, Waterman AD, Ebers AG, et al. Patients' and physicians' attitudes regarding the disclosure of medical errors. JAMA 2003; 289: 1001-1007.

5 Studdert DM. Disclosure of medical injury. In: Healy J, Dugdale P, editors. Patient safety first: responsive regulation in health care. Sydney: Allen and Unwin, 2009.

6 Australian Council for Safety and Quality in Health Care. Open Disclosure Standard: a national standard for open communication in public and private hospitals, following an adverse event in health care. Sydney: ACSOHC, 2003. http://www.safetyandquality.gov.au/internet/safety/publishing.nsf/Content/PriorityProgram-02 (accessed May 2010).

7 ledema R, Mallock NA, Sorenson RJ, et al. The National Open Disclosure Pilot: evaluation of a policy implementation initiative. Med J Aust 2008; 188: 397-400.

8 Lamb RM, Studdert DM, Bohmer RMJ, et al. Hospital disclosure practices: results of a national survey. Health Aff (Millwood) 2003; 22: 73-83.
9 Gibson R, Singh JP. Wall of silence: the untold story of the medical mistakes that kill and injure millions of Americans. Washington, DC: Lifeline Press, 2003.

10 Constas MA. Qualitative analysis as a public event: the documentation of category development procedures. Am Educ Res J 1992; 29: 253266.

11 Studdert DM, Richardson MW. Legal aspects of open disclosure: a review of Australian law. Med J Aust 2010; 193: 273-276.

12 Australian Commission on Safety and Quality in Health Care. Windows into safety and quality in health care 2008. Sydney: ACSOHC, 2008. http://www.health.gov.au/internet/safety/publishing.nsf/Content/E060D889E298D039CA 2574EF00721BD8/\$File/ACSQHC_National\% 20Report.pdf (accessed Oct 2009).

13 ledema R, Mallock N, Sorensen R, et al. Evaluation of the pilot of the National Open Disclosure Standard. Final report. Sydney: University of Technology Sydney, 2007. http:// www.health.gov.au/internet/safety/publishing.nsf/Content/751691A67FE79CFECA2577 6000155EE9/\$File/EvaluationOf-Pilot-NODStd.PDF (accessed Oct 2009).

14 New South Wales Health. Open disclosure: training and education. http://www.health.nsw. gov.au/quality/opendisc/training.asp (accessed Oct 2009).

15 ledema R, Jorm C, Wakefield J, et al. Practising open disclosure: clinical incident communication and systems improvement. Sociol Health IIIn 2009; 31: 262-277.

16 Studdert DM, Brennan TA. No-fault compensation for medical injuries: the prospect for error prevention. JAMA 2001; 286: 217-223.

17 Mello MM, Studdert DM, Kachalia A, Brennan TA. Health courts and accountability for patient safety. Milbank Q 2006; 84: 459-482.

18 Kachalia A, Kaufman SR, Boothman R, et al. Liability claims and costs before and after implementation of a medical error disclosure program. Ann Intern Med 2010; 153: 213-221.

19 Ferlie EB, Shortell SM. Improving the quality of health care in the United Kingdom and the United States: a framework for change. Milbank Q 2001; 79: 281-315.

20 Gallagher TH, Waterman AD, Garbutt JM, et al. US and Canadian physicians' attitudes and experiences regarding disclosing errors to patients. Arch Intern Med 2006; 166: 1605-1611.

21 Berlinger N. After harm: medical error and the ethics of forgiveness. Baltimore: Johns Hopkins University Press, 1995.

(Received 4 Feb 2010, accepted 31 May 2010) 